\title{
Transplantation of cryopreserved human umbilical cord blood-derived endothelial progenitor cells induces recovery of carotid artery injury in nude rats
}

Yangguang Yin ${ }^{1+}$, Huanyun Liü ${ }^{2+}$, Fangjuan Wang ${ }^{2}$, Lufeng $\mathrm{Li}^{2}$, Mengyang Deng ${ }^{2}$, Lan Huang ${ }^{2}$ and Xiaohui Zhao ${ }^{2 *}$

\begin{abstract}
Introduction: Transplantation of endothelial progenitor cells (EPCS) restores endothelial function in patients with endothelial dysfunction and initial denudation. The goal of the present study was to determine the effect of cryopreserved human umbilical cord blood (UCB)-derived EPC infusion on the repair of carotid artery injury in nude rats.
\end{abstract}

Methods: Mononuclear cells (MNCS) from human cryopreserved UCB and peripheral blood (PB) of patients with cardiovascular diseases and healthy volunteers were cultured in a conditioned medium. The in vitro migration, proliferation, adhesion, and survival capacities, as well as paracrine cytokine release of EPCs were investigated. EPC homing, induced reendothelialization, and the effect on neointima formation were also assessed in vivo.

Results: Patient-derived PB EPCS (PPB-EPCs) displayed decreased migration, proliferation, adhesion, and survival capabilities as compared to PB-EPCs from healthy volunteers (HPB-EPCs) and cryopreserved UCB-EPCs. However, there was no difference in the release of vascular endothelial growth factor (VEGF) and stromal cell derived factor 1 (SDF-1) between the three groups. Two weeks after transplantation, more labeled UCB-EPCs and HPB-EPCs than PPB-EPCs were found by cell tracking in the injury zone. Administration of PPB-EPCs, HPB-EPCs, and UCB-EPCs enhanced reendothelialization and inhibited neointima formation compared to the saline control. However, UCB-EPC and HPB-EPC infusion showed a greater improvement than PPB-EPCs.

Conclusions: Cryopreserved UCB-MNCs derived EPCS and HPB-EPCS show better responses to cytokines and vascular injury than PPB-EPCs. Thus, cryopreservation and delivery of cryopreserved autogenous UCB-EPCs or HPB-EPCs may be a promising vasculoprotective approach for patients with multiple cardiovascular risk factors.

\section{Introduction}

Endothelial dysfunction and initial denudation are major contributing factors to vasoconstriction, neointima formation, thrombosis, and atherosclerosis [1]. Previous experiments have suggested that endothelial progenitor cells (EPCs) - derived from hematopoietic stem cells (HSCs) - have the potential to incorporate into the site of vessel injury and differentiate into endothelial cells, thereby contributing to the improvement of endothelial function [2]. Transplantation of

\footnotetext{
* Correspondence: zxhwn@tmmu.edu.cn

${ }^{\dagger}$ Equal contributors

${ }^{2}$ Cardiovascular Disease Research Center, Xinqiao Hospital, Third Military

Medical University, Chongqing 400037, China

Full list of author information is available at the end of the article
}

EPCs is currently under intensive investigation and has proven to be a useful strategy in animal models and clinical research [3]. However, growing evidence has shown that EPCs from patients with cardiovascular risk factors, including diabetes, hypertension, metabolic syndrome, smoking, aging, and coronary artery disease, or other diseases, such as emphysema, acute lung injury, liver fibrosis, and systemic sclerosis, are associated with decreased number and impaired function $[4,5]$.

Umbilical cord blood (UCB) is a traditional source of HSCs for the treatment of various diseases beyond hematologic diseases such as Langerhans-cell histocytosis and Bare-lymphocyte syndrome. UCB has been shown to contain a large number of progenitors, and transplantation 
of these cells can lead to reendothelialization of denuded vessels by both directly differentiating into endothelial cells and the release of paracrine factors [6]. In contrast to adult EPCs, cord blood EPCs have a higher proliferative capacity, rapid self-renewal, low apoptosis, and express telomerase, a functional characteristic of stem cells that is very low or absent in other progenitor cell populations [7,8]. All of these properties favor the exciting opportunity to obtain a large quantity and high quality UCB-EPCs, compared with peripheral blood (PB). Furthermore, UCB stem cells can be extracted and cryopreserved, allowing for future personal autotransplantation or matchedpatient use. Previous studies have shown that cryopreserved UCB-mononuclear cells (MNCs) can differentiate into EPCs in conditioned medium, and exhibit similar properties to those of fresh UCB in vitro and in vivo [9-11].

In this study, we compared the effect of cryopreserved human UCB-derived EPCs, PB-derived EPCs from patients with cardiovascular risk factors (PPB-EPCs), and PB derived EPCs of healthy volunteers (HPB-EPCs) on the repair of carotid artery injury in nude rats.

\section{Methods}

\section{Human peripheral blood-mononuclear cells}

All experiments in this study were conducted in accordance with the Position of the American Heart Association on Research and Animal Use adopted by the American Heart Association and the guidelines of the Institutional Animal Care and Use Committee of Third Military Medical University. Written informed consent was obtained from all patients and normal subjects before enrollment in the study. All aspects that involved human or human tissue in our study were approved by Committees of Ethics at Third Military Medical University. Patients with cardiovascular diseases $(n=25$, Table 1$)$ and healthy volunteers $(n=25$, Table 1$)$ were included in the study. MNCs were isolated by density gradient centrifugation using a Histopaque density centrifugation method (Sigma, St. Louis, MO, USA) from $20 \mathrm{~mL}$ of PB.

Table 1 Patients and healthy volunteer characteristics

\begin{tabular}{lll}
\hline Characteristics & $\begin{array}{l}\text { Patients } \\
\text { (number = 25) }\end{array}$ & $\begin{array}{l}\text { Healthy volunteers } \\
\text { (number = 25) }\end{array}$ \\
\hline Age (years) & $64.04 \pm 11.13$ & $62.87 \pm 9.75$ \\
Sex (men) & 18 & 15 \\
Smokers & 13 & 0 \\
LDL $>130 \mathrm{mg} / \mathrm{dL}$ & 7 & 0 \\
Coronary artery disease & 18 & 0 \\
Diabetes & 4 & 0 \\
Hypertension & 14 & 0 \\
\hline
\end{tabular}

\section{UCB-MNC freezing and thawing}

UCB was collected from umbilical veins after neonatal delivery by a cord blood bank (Sichuan province, China) with informed consent from pregnant mothers. Freezing and thawing of UCB-MNCs was performed according to previously described methods $[9,10]$. Briefly, human UCB samples (40 to $90 \mathrm{~mL}$ ) from female full-term newborns were collected from a fresh umbilical cord attached to the placenta and MNCs were isolated from the collected blood using the Histopaque density centrifugation method. The collected MNC layers from UCB were washed three times with phosphate-buffered saline (PBS, Sigma) and suspended at $5 \times 10^{7}$ cells $/ \mathrm{mL}$ with a final concentration of $10 \%$ dimethyl sulfoxide (DMSO) (Sigma) and 20\% fetal bovine serum (FBS) (Sigma). Then the cryotubes were transferred to a programmed cryopreservation box and stored in liquid nitrogen $\left(-196^{\circ} \mathrm{C}\right)$. UCB samples that had been in cryostorage for two to five years were used for this study.

The cryotubes were thawed very rapidly in a $37^{\circ} \mathrm{C}$ water bath and the MNCs were immediately transferred to $50 \mathrm{~mL}$ thawing medium containing $5 \% \mathrm{FBS}+$ DNase I ( $20 \mathrm{U} / \mathrm{mL}$, Roche, Shanghai, China). Then, the MNCs were centrifuged at $300 \times g$ for 15 minutes at room temperature. The supernatant was removed and then MNCs were resuspended and plated on fibronectin-coated 24-well plates $\left(1 \times 10^{6} / \mathrm{cm}^{2}\right.$, Sigma) in $0.5 \mathrm{~mL}$ Dulbecco's modified Eagle's medium (DMEM, Gibco, Carlsbad, CA, USA) supplemented with 20\% FBS.

\section{EPC culture}

EPCs were cultured according to previously described techniques [12]. Briefly, MNCs were plated on fibronectincoated 24-well plates $\left(1 \times 10^{6} / \mathrm{cm}^{2}\right)$ in $0.5 \mathrm{~mL}$ DMEM supplemented with $20 \% \mathrm{FBS}$, vascular endothelial growth factor (VEGF, $50 \mathrm{ng} / \mathrm{mL}$ ), basic fibroblast growth factor (bFGF, $5 \mathrm{ng} / \mathrm{mL}$ ), epidermal growth factor (EGF, $10 \mathrm{ng} / \mathrm{mL}$ ), penicillin $(100 \mathrm{U} / \mathrm{mL})$, and streptomycin $(100 \mu \mathrm{g} / \mathrm{mL})$. Four days after culturing, nonadherent cells were removed by thorough washing with PBS. The medium was changed every three days.

\section{EPC characterization}

EPCs were characterized according to previously described techniques [13]. After seven days, cells were incubated with $2.4 \mathrm{ng} / \mathrm{mL}$ DiI-labeled acetylated low-density lipoprotein (DiI-LDL, Molecular Probes, Waltham, MA, USA) at $37^{\circ} \mathrm{C}$ for one hour. Then the cells were washed with PBS three times and fixed with 2\% paraformaldehyde (PFA, Beijing CellChip Biotechnology, Beijing, China) for 10 minutes. After washing with PBS, the cells were stained with a fluorescein isothiocyanate (FITC)-labeled lectin from ulex europaeus agglutinin (UEA, Vector Labs, Burlingame, CA, USA) for one hour. Samples were washed with 
PBS and viewed with an inverted fluorescent microscope (Olympus, Tokyo, Japan). Double-labeled fluorescence cells were identified as differentiating EPCs. The negative control was incubated with PBS instead of DiI-LDL and UEA.

The cells were labeled with $1 \mathrm{mg} / \mathrm{L}$ FITC or phycoerythrin (PE)-conjugated mouse antihuman CD31-PE (Beckman Coulter, Paris, France), CD45-FITC, CD34FITC, and VEGFR2-PE (BD Biosciences, Franklin Lakes, NJ, USA) monoclonal antibodies for 30 minutes at $4^{\circ} \mathrm{C}$ in PBS. FITC- or PE-conjugated mouse immunoglobulin G (IgG) was used as an isotype control at the same concentration. The fluorescence intensity of the cells was evaluated by flow cytometry using a flow cytometer (BD Biosciences), and the data were analyzed with CellQuest software (BD Biosciences).

Human umbilical venous endothelial cells (HUVECs) isolated and pooled from umbilical cords obtained from normal vaginal deliveries (Cell Applications, San Diego, CA, USA) were passaged three times and used as the positive control.

\section{EPC migration assay}

EPC migration was evaluated using a modified Boyden chamber (Chemicon, Temecula, CA, USA) as previously described [14]. Stromal cell derived factor-1 (SDF-1, Sigma) was diluted to $100 \mathrm{ng} / \mathrm{mL}$ in DMEM and $25 \mu \mathrm{L}$ of the final dilution was placed in the lower chamber. EPCs $\left(2.5 \times 10^{5}\right)$ suspended in $50 \mu \mathrm{L}$ DMEM were seeded in the upper compartment. After six hours incubation at $37^{\circ} \mathrm{C}$, the lower side of the filter was washed with PBS and fixed with 2\% PFA. For quantification, cells were stained with Giemsa solution (Sagene, Guangzhou, China). Cells that had migrated into the lower chamber were counted manually in five random high-power fields $(n=9)$. Control experiments were performed by stimulation with PBS. The experiments were repeated three times.

\section{EPC proliferation assay}

The proliferation assay was performed as previously described [12]. Briefly, EPCs were digested with $0.25 \%$ trypsin (Sagene) and then seeded in 96-well culture plates (Sigma). After overnight culture in phenol red-free DMEM, cells were incubated with $100 \mathrm{ng} / \mathrm{mL}$ rhVEGF (ProSpec, East Brunswick, NJ, USA) for 24 hours. Before the optical density measurement $(562 \mathrm{~nm}$, Tecan, Zurich, Switzerland) was performed, EPCs were supplemented with $10 \mu \mathrm{L}$ MTT (5 g/L, Gibco) for another six hours and $200 \mu \mathrm{L}$ DMSO for 10 minutes.

Mitogenic activity was further evaluated by cell counting. EPCs were seeded in six-well plates (Sigma) at a density of $5 \times 10^{5}$ cells per well in phenol red-free DMEM overnight, and then exposed to $100 \mathrm{ng} / \mathrm{mL}$ rhVEGF for another 24 hours.

\section{Cell adhesion assay}

The cell adhesion assay was performed as previously described [14]. Briefly, EPCs were incubated with SDF-1 $\alpha$ (100 ng/mL) for 12 hours and detached as described above. After centrifugation and resuspension in EBM (endothelial basal medium) (Cell Systems, Kirkland, WA, USA) with $5 \%(\mathrm{v} / \mathrm{v})$ FBS, identical cells were plated onto fibronectin-coated culture dishes and incubated for 30 minutes at $37^{\circ} \mathrm{C}$. Adherent cells were counted in three random microscopic fields $(\times 400)$.

\section{Apoptosis assay}

Apoptosis assays were carried out using an annexin $\mathrm{V}$ apoptosis detection kit (Alexis Biochemicals, Farmingdale, NY, USA), according to the manufacturer's instructions [15]. EPCs were incubated with $50 \mathrm{mg} / \mathrm{L}$ TNF- $\alpha$ (Sigma) for four days and then stained with annexin $\mathrm{V}$ FITC and propidium iodide (PI). To exclude necrotic cells, only annexin-positive cells were subjected to flow cytometric analysis. Data are provided as the mean (SEM) of the percentage of annexin $\mathrm{V}^{+} / \mathrm{PI}^{-}$cells (apoptotic cells).

\section{Western blot analysis}

Cells were incubated with lysis buffer (Sagene) for 20 minutes at $4^{\circ} \mathrm{C}$. After centrifugation for 15 minutes at $20,000 \times g\left(4^{\circ} \mathrm{C}\right)$, the protein concentration was quantified for each sample and $50 \mu \mathrm{g}$ per lane was loaded onto SDS-polyacrylamide gels (Pharmacia Biotech, Buckinghamshire, UK). The separated proteins were electrotransferred to nitrocellulose membranes (Pharmacia Biotech) and probed with antibodies against VEGF and SDF-1 (1:1000 dilution, R\&D Systems, Minneapolis, $\mathrm{MN}$, USA). The membranes were then incubated with a horseradish peroxidase (HRP)-conjugated secondary antibody (R\&D Systems, 1:2000) for two hours, and reacted with the chromogenic substrate 3,3'-diaminobenzidine (DAB, Sigma). Blots were reprobed with GAPDH (1:2000, Sigma). The autoradiographs were scanned and analyzed semi-quantitatively.

\section{Enzyme-linked immunosorbent assay}

ELISAs were performed according to the manufacturer's instructions ( $\& \& D$ Systems). Briefly, $1 \times 10^{6}$ cells were incubated with $1 \mathrm{~mL}$ DMEM without supplements for 24 hours. The medium was collected and $20 \times$ concentrated by centrifugation at $4^{\circ} \mathrm{C}(5000 \times g$ for 20 minutes $)$ using Ultrafree-4 centrifugal filter tubes with Biomax-5 membranes (Millipore, Billerica, MA, USA). VEGF and SDF-1 were then measured in concentrated culture supernatants by ELISA. 


\section{Carotid artery injury and EPC transplantation}

Carotid artery injury and EPC transplantation was performed using previously described methods [16]. Heparin (Wanbang, Shanghai, China) was intraperitoneally administered at a dosage of $300 \mathrm{U} / \mathrm{kg}$. After 30 minutes, six-week-old female nude rats (Laboratory Animal Department of Shanghai Public Health Clinical Center) were anesthetized with $50 \mathrm{mg} / \mathrm{kg}$ sodium pentobarbital (Xinhua, China) injected intraperitoneally. Left carotid arteries were exposed through a midline incision in the neck. Silk sutures (6-0, Suzhou Medical Appliance Factory, Suzhou, China) were placed around the common, internal, and external carotid arteries to temporarily restrict blood flow to the area of surgical manipulation. A 1.5 F Fogarty arterial embolectomy catheter (Baxter, Deerfield, IL, USA) was inserted through the external carotid artery, inflated with $150 \mu \mathrm{L}$ of air, and passed three times along the length of the segment. The total length of denuded common carotid artery was $10 \mathrm{~mm}$ from the bifurcation of carotid arteries in all animals. Then the external carotid artery was permanently ligated. The temporary ligatures were released to allow blood flow to be restored through the internal carotid artery. The skin was closed with single sutures using 6-0 silk. Animals were euthanized and carotid arteries were harvested at indicated time points after induction of carotid artery injury.

Labeled EPCs $\left(1 \times 10^{6}\right)$ were incubated with $2.4 \mu \mathrm{g} / \mathrm{mL}$ DiI-LDL (Molecular Probes) for one hour. Then the cells were washed with PBS three times and injected into the rats' tail vein in $200 \mu \mathrm{L}$ saline after induction of arterial injury.

\section{Assessment of reendothelialization}

Assessment of reendothelialization was performed according to previously described methods [13]. One week after carotid artery injury and labeled EPC transplantation, $200 \mu \mathrm{L}$ of $5 \%$ Evans blue (Sigma) was injected into the heart with a 27-gauge needle (Suzhou Medical Appliance Factory). Then 4\% PFA (Sigma) was administered for perfusion fixation. The remaining endothelium-denuded site(s) within the harvested arterial segment was measured using Image-Pro Plus 5.1 (Media Cybernetics Inc., Bethesda, MD, USA) by one analyst, who was blinded to the treatment regimen. The initially denuded area was defined as the total surface area of the harvested arterial segment. The length of the harvested segment corresponded to the total length of the injured segment; in each case, this length was similarly defined proximally by the carotid bifurcation and distally by the edge of the omohyoid muscle. The reendothelialized area was defined macroscopically as the area that was not stained with Evans blue dye. In addition to Evans blue staining, scanning electron microscopy was performed to evaluate endothelial recovery and morphology. Samples for electron microscopy were fixed and processed using a standard technique.

\section{Measurement of medial and neointimal area}

Measurement of the medial and neointimal areas was performed according to previously described methods [13]. Three weeks after carotid artery injury, the left common carotid artery was harvested $10 \mathrm{~mm}$ from the carotid bifurcation. After perfusion fixation with 2\% PFA and paraffin-embedding, carotid cross-sections $(5 \mu \mathrm{m})$ were stained with hematoxylin and eosin (Boyao Bio, Shanghai, China) and Verhoeff van Geison (GenMed, Shanghai, China) according to standard protocols. All sections were examined under an inverted microscope (Leica, Wetzlar, Germany). Morphometric analysis of the medial and neointimal area was performed with Image-Pro Plus 5.1 (Media Cybernetics Inc., Bethesda, MD, USA). Endoluminal, internal elastic laminar, and external elastic laminar borders were manually traced with the software (Image ProPlus) to calculate the intimal and medial areas of 15 sections per animal.

\section{Statistical analysis}

All values were expressed as the mean \pm standard deviation (SD). Differences between data were analyzed with analysis of variance (ANOVA) followed by Fisher's Least Significant Difference (LSD) using SPSS19.0. $P<0.05$ was considered to be statistically significant.

\section{Results}

Characterization of cultured EPCs

Cryopreserved UCB-MNCs cultured under endothelialspecific conditions developed into normal cell colonies by day 7 after isolation (Figure 1A). The center cells in the clones showed less incorporation of DiI-LDL (red, Figure 1B) and endothelial-specific lectin (green, Figure $1 \mathrm{C}$ ), which indicated that the outer cells were differentiating endothelial cells.

Adherent cells from cryopreserved-derived UCB-MNCs intensely bound endothelial-specific lectin (green, Figure 1D) and took up acetylated LDL (red, Figure 1E). Doublepositive cultured MNCs (yellow, $90.02 \pm 7.74 \%$, Figure $1 \mathrm{~F}$, $\mathrm{n}=5$ ) indicated endothelial function. PPB- and HPB-EPCs showed $90.9 \pm 11.14 \%$ and $91.04 \pm 8.76 \%$ double staining for DiI and UEA, respectively [see Additional file 1].

DiI-LDL-labeled EPCs were placed in Matrigel-based media in which they rapidly formed capillary-like structures (Figure $1 \mathrm{G}$ and $\mathrm{H}$ ). The identity of EPCs was additionally confirmed by the detection of multiple dense round or rod-shaped structures (Weibel-Palade bodies) in the electron micrographs (Figure 1I). 

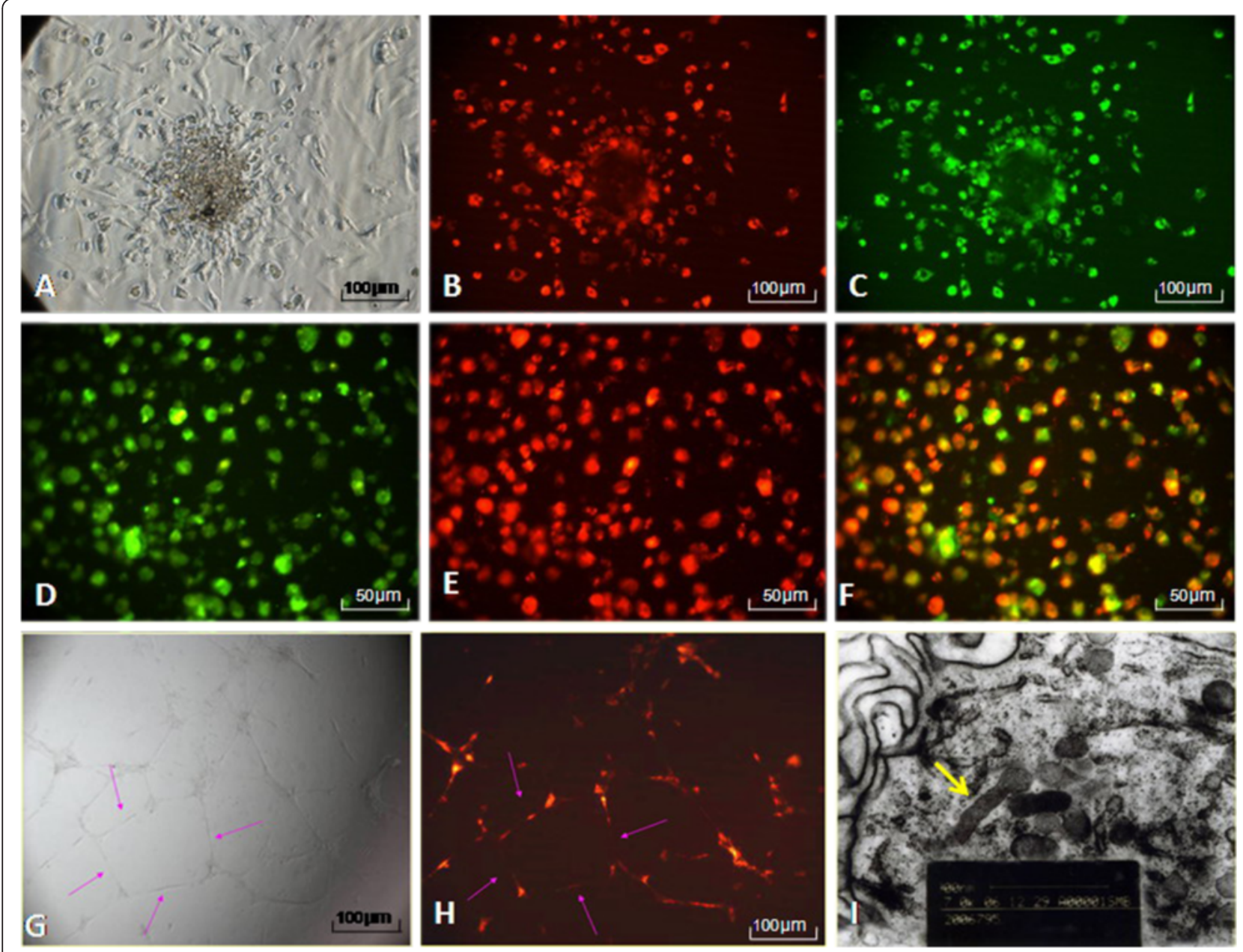

Figure 1 Cryopreserved umbilical cord blood mononuclear cells (UCB-MNCs) differentiated into endothelial progenitor cells (EPCs) under endothelial cell-specific culture conditions. Culture of MNCs resulted in the emergence of colonies (A, B, and C). Cells double-labeled for fluorescein isothiocyanate (FITC)-lectin binding (green, D) and Dil-labeled acetylated low-density lipoprotein (Dil-LDL) uptake (red, E) were identified as EPCS (yellow, F). Tube formation assay ( $\mathbf{G}$ and $\mathbf{H}$, red arrow) indicated endothelial function. Transmission electron microscope image of cultured cells indicates the existence of Weibel-Palade bodies, a typical superstructure of endothelial cells (I, yellow arrow)

Furthermore, flow cytometry analysis (Figure 2) was performed to evaluate the immunophenotype of the cryopreserved-derived MNCs (Figure $2 \mathrm{~A}$ ) on day 0 (before differentiation) and day 7. We found increased expression of endothelial markers (CD31, CD34, and VEGFR2) and decreased expression of hematopoietic stem cell markers (CD45) on day 7 , indicating that the cultured MNCs were differentiating into endothelial cells. PPB- and HPB-MNCs (Figure $2 \mathrm{~B}$ and C) were also examined on day 0 and 7 . Fully-differentiated HUVECs were used as positive controls (Figure 2D).

\section{EPC migration activity evaluation}

Directed migratory responses of EPCs to SDF-1 were performed using a modified Boyden chamber. Stimulation with $100 \mathrm{ng} / \mathrm{mL}$ SDF-1 induced a significant increase in migration of the PPB group (PPB versus control,
$P<0.05)$, HPB group (HPB versus control, $P<0.001$ ), and cryopreserved UCB group (UCB versus control, $P<0.001$ ). Furthermore, there were more migrated EPCs in the cryopreserved UCB group and HPB group than in the PPB group (UCB versus $\mathrm{PPB}, P<0.01$; HPB versus $\mathrm{PPB}, P<0.05)$ (Figure 3A). There was no difference between the cryopreserved UCB group and the HPB group.

\section{EPC proliferation activity evaluation}

The proliferative response of EPCs to VEGF was evaluated using MTT tetrazolium dye viability assays. Stimulation with $100 \mathrm{ng} / \mathrm{mL}$ VEGF showed a significant increase in proliferation of the PPB group (PPB versus control, $P<0.01$ ), HPB group (HPB versus control, $P<0.001$ ), and cryopreserved UCB group (UCB versus control, $P<0.001)$. In addition, cryopreserved UCB-EPCs and HPB-EPCs displayed stronger proliferation than PPB-EPCs 


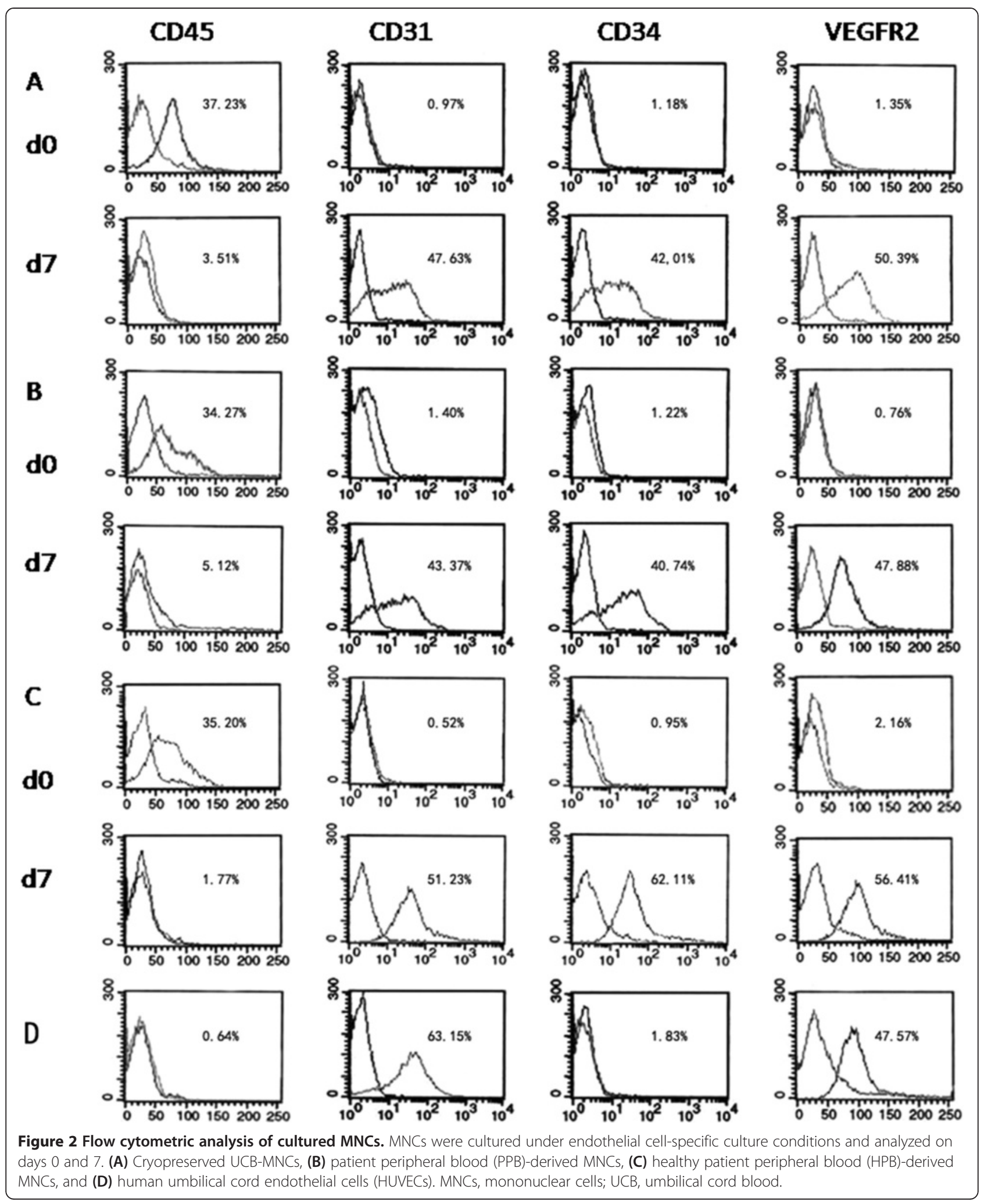

(UCB versus $\mathrm{PPB}, P<0.05$; $\mathrm{HPB}$ versus $\mathrm{PPB}, P<0.01$ ) (Figure $3 \mathrm{~B}$ ). There was still no difference between the cryopreserved UCB group and the HPB group.
The increase in VEGF-induced proliferation activity was confirmed by manual counting of EPCs in the PPB group (PPB versus control, $P<0.05$ ), HPB group (HPB 

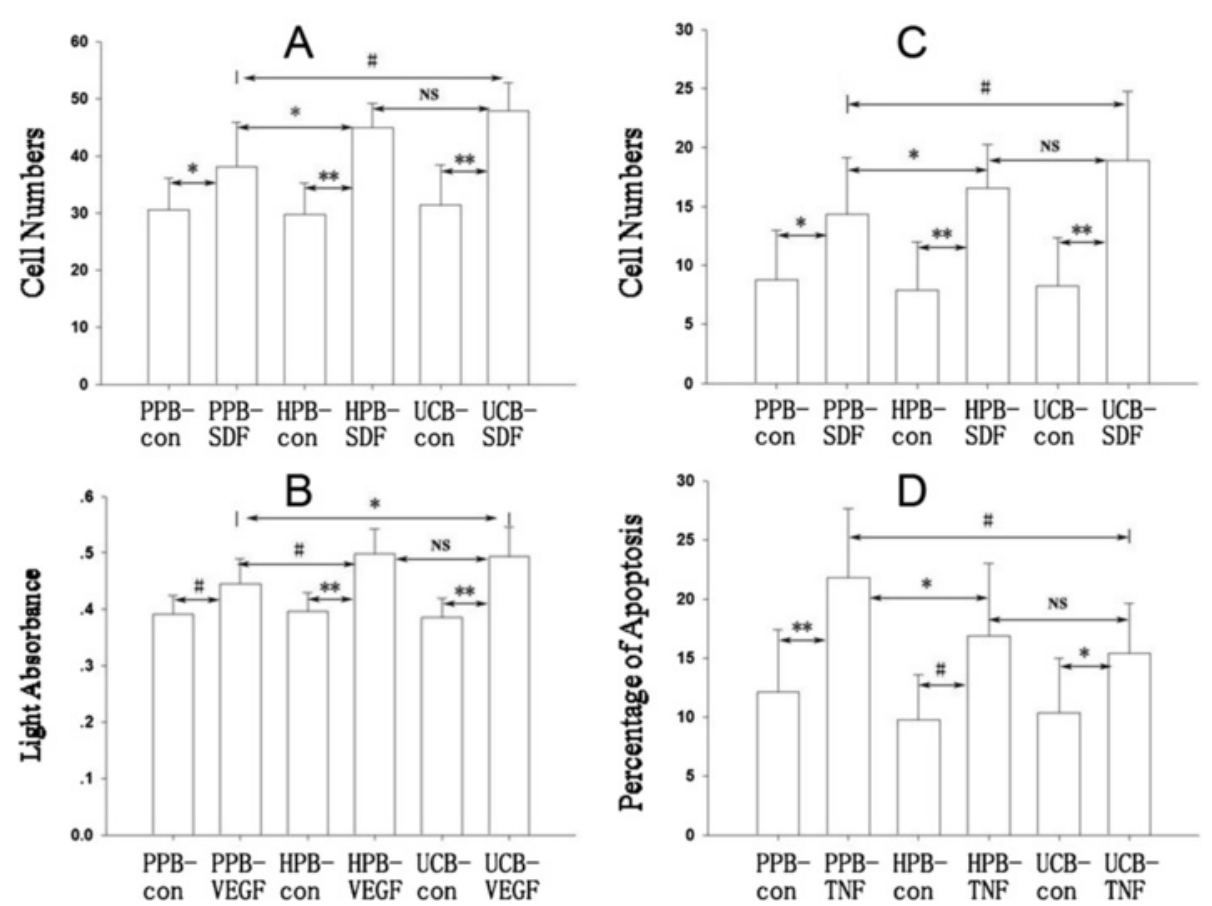

Figure 3 Evaluation of EPC biological function in vitro. (A) Migration of EPCS cultured with $100 \mathrm{ng} / \mathrm{mL} S D F-1, n=9$. (B) Proliferation of EPCs cultured with $100 \mathrm{ng} / \mathrm{mL}$ VEGF, $\mathrm{n}=9$. (C) Adhesion test with $100 \mathrm{ng} / \mathrm{mL}$ SDF-1, $\mathrm{n}=9$. (D) Apoptosis test with $100 \mathrm{mg} / \mathrm{L}$ TNF-a, $n=9$. PPB-control, PPB-EPCs treated with PBS; HPB-control, HPB-EPCs treated with phosphate buffered saline (PBS); UCB-control, UCB-EPCs treated with PBS. * $P<0.05$; $\# P<0.01$, ** $P<0.001$; NS, not significant. EPC, endothelial progenitor cells; HPB, peripheral blood from healthy volunteers; PPB, peripheral blood from patients; SDF-1, stromal cell derived factor 1; UBC, umbilical cord blood; VEGF, vascular endothelial growth factor.

versus control, $P<0.01$ ), and UCB group (UCB vrsus control, $P<0.001)$. The number of cells in the UCB and HPB groups was significantly greater than that of the PPB group (UCB versus PPB, $P<0.01$; HPB versus PPB, $P<0.05) \times 10^{5} /$ well [see Additional file 2]. No difference was seen between the cryopreserved UCB group and the HPB group.

\section{Cell adhesion assay}

Incubation of EPCs with $100 \mathrm{ng} / \mathrm{mL}$ SDF- $1 \alpha$ significantly increased the number of adherent cells (PPB versus control, $P<0.05$; HPB versus control, $P<0.001$; UCB versus control, $P<0.001)$. There was also a significant increase in the overall number of cells in the UCB and HPB groups compared to the PPB group (UCB versus PPB, $P<0.01$; HPB versu. $\mathrm{PPB}, P<0.05$; UCB versus $\mathrm{HPB}, P=\mathrm{NS}$ ) (Figure $3 \mathrm{C}$ ).

\section{Apoptosis assay}

EPCs cultured with $100 \mathrm{mg} / \mathrm{L}$ tumor necrosis factor-alpha (TNF- $\alpha$ ) for four days showed a significant increase in the number of apoptosis cells in the PPB group (PPB versus control, $P<0.001$ ), HPB group (HPB versus control, $P<0.01$ ), and UCB group (UCB versus control, $P<0.05$ ). There were significantly fewer apoptotic EPCs in the UCB group and $\mathrm{HPB}$ groups than the PPB group (UCB versus
PPB, $P<0.01$; HPB versus PPB, $P<0.05$; UCB versus HPB, $P=\mathrm{NS}$ ) (Figure 3D).

\section{Cytokine release from EPCs in vitro}

We measured the release of different cytokines involved in the regulation of EPC function. As shown in Figure 4, the release of VEGF and SDF-1 were significantly increased in UCB-EPCs, PPB-EPCs, and HPB-EPCs compared to MNCs and HUVECs (all $P<0.001$ ). However, there was no difference among the UCB, PPB, and HPB groups (all $P>0.05$ ).

\section{Transplantation of cryopreserved EPCs promoted reendothelialization}

To determine the effect of EPC transplantation on endothelium recovery, rats were injected with Evans blue seven days after carotid artery injury. The blue area was considered the denuded zone. Reendothelialization is represented by the ratio of reendothelialized area (white)/ total area (white + blue). Endothelial cell regeneration was significantly enhanced in the PPB-EPC, HPB-EPC, and cryopreserved UCB-EPC treated vessels compared to the saline injection control (PPB versus control, $P<0.05$; HPB versus control, $P<0.001$; UCB versus control, $P<0.001$; Figure $5 \mathrm{~A}$ and $\mathrm{B}, \mathrm{n}=5$ ). Furthermore, the cryopreserved UCB-EPCs and HPB-EPCs displayed improved endothelial 

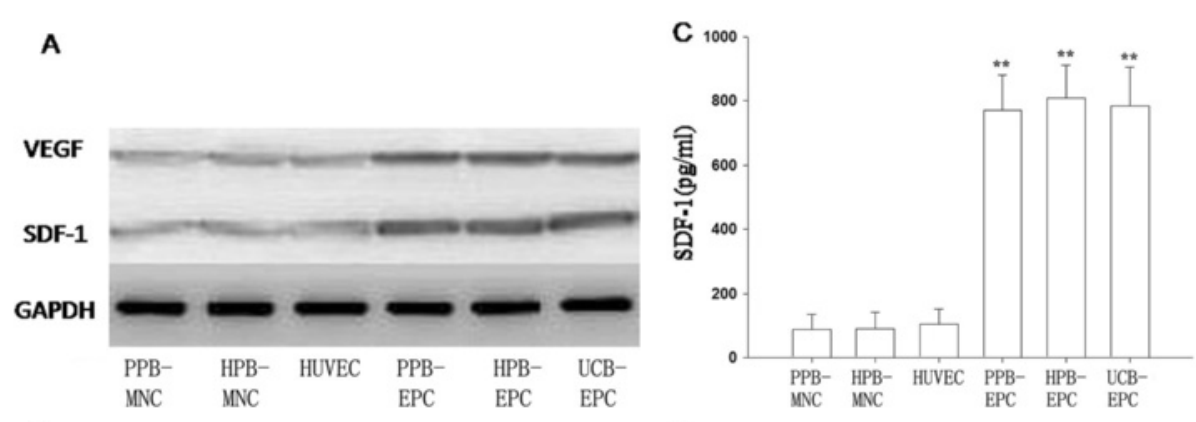

B

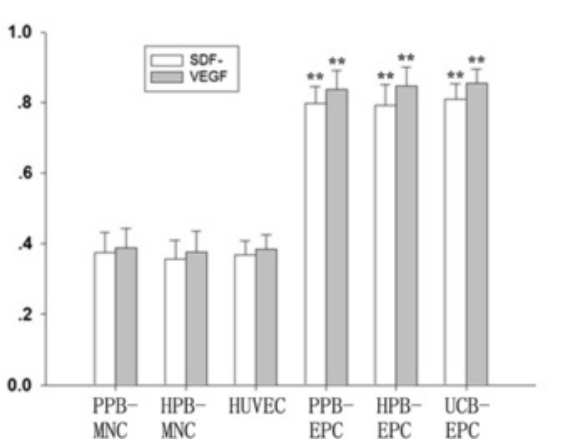

D

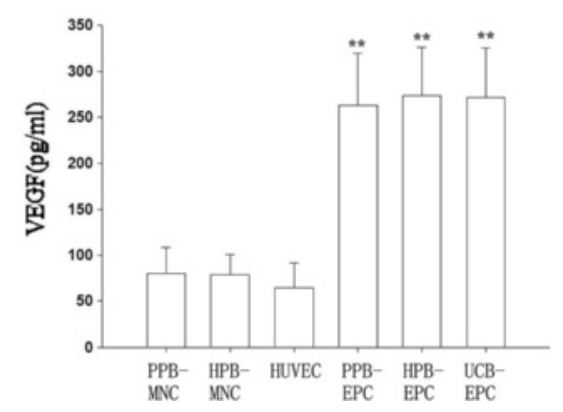

Figure 4 Increased protein expression of vascular endothelial growth factor (VEGF) and stromal cell-derived growth factor 1 (SDF-1) in EPCs compared to mature endothelial cells and MNCs. (A) A representative western blot of three independent experiments. GAPDH serves as the loading control. (B) Protein expression quantified by densitometry analysis. The ratio for VEGF or SDF-1 loading is shown. (C and D) HUVECS, EPCs, and MNCs were incubated with equal amounts of fresh medium for 24 hours. The medium was collected, 20x concentrated, and measured by ELISA, $\mathrm{n} \geq 6,{ }^{* *} P<0.001$ versus HUVECs and MNCs. EPCs, endothelial progenitor cells; HUVECs, human umbilical venous endothelial cells;

MNCs, mononuclear cells.

cell regeneration compared to the PPB-EPC injection (UCB versus PPB, $P<0.001$; $\mathrm{HPB}$ versus $\mathrm{PPB}, P<0.01$; Figure $5 \mathrm{~A}$ and $\mathrm{B}, \mathrm{n}=5$ ). More endothelial cell regeneration was also seen in the UCB group than the HPB group (UCB versus $\mathrm{HPB}, P<0.05$, Figure $5 \mathrm{~A}$ and $\mathrm{B}$ ). Normal carotid artery was used as the negative control and injured artery (day 0) was used as the positive control [see Additional file 3]. Scanning electron microscopy analysis revealed characteristic features of regenerated endothelium derived from EPCs. The regenerated endothelium appeared rough, whereas the surface of the normal endothelium was smooth and covered with a monolayer coat (Figure 5D).

\section{Labeled EPCs are detectable in injured artery}

To determine whether labeled EPCs incorporated into the injured vessel wall and differentiated into endothelial cells, animals received $1 \times 10^{6}$ DiI-labeled EPCs by tail vein injection after carotid artery injury. Two weeks later, EPC tracking and immunohistochemistry were performed. DiI-labeled EPCs were identified as red fluorescence cells (Figure 5E). Labeled cells were seen lining the lumen that co-stained for endothelial markers FITC-UEA. No DiI-labeled cells were identified in uninjured control arteries (data not shown). However, there were UEA-positive cells within the neointima, indicating in situ endothelial cells (data not shown). Furthermore, there were more homing EPCs in the cryopreserved UCB and HPB groups than in the PPB group (Figure $5 \mathrm{C}$, all $P<0.05)$.

\section{Transplantation of cryopreserved EPCs inhibited neointima}

Only monolayer endothelial cells were seen along the inner lumina of the normal carotid artery, which indicated that there was no evidence of neointima in the sham group. However, balloon injury resulted in prominent neointima (Figure 6A, black arrows). Transplantation of cryopreserved UCB-EPCs, PPB-EPCs, and HPB-EPCs inhibited neointima development in the injured vessels (PPB versus control, $P<0.05$; HPB versus control, $P<0.001$; UCB versus control, $P<0.001$, Figure $6 \mathrm{~B}$, $\left.\mathrm{n}=4, \times 10^{4} \mu \mathrm{m}^{2}\right)$. Cryopreserved UCB-EPC and HPB-EPC infusion displayed an improved effect compared to the PPB-EPCs $(P<0.01$ or $P<0.05)$. There was no difference in the media area among the four groups (Figure $6 \mathrm{C}$ ). Compared with the saline control, morphometric analysis showed a decrease in the neointima/media ratio in EPC-transplanted rats (PPB versus control, $P<0.05$, HPB versus control, $P<0.001$; UCB versus control, $P<0.001$, Figure $6 \mathrm{D}, \mathrm{n}=4)$. UCB-EPC and HPB-EPC infusion also displayed an improved effect compared to the PPB-EPCs 


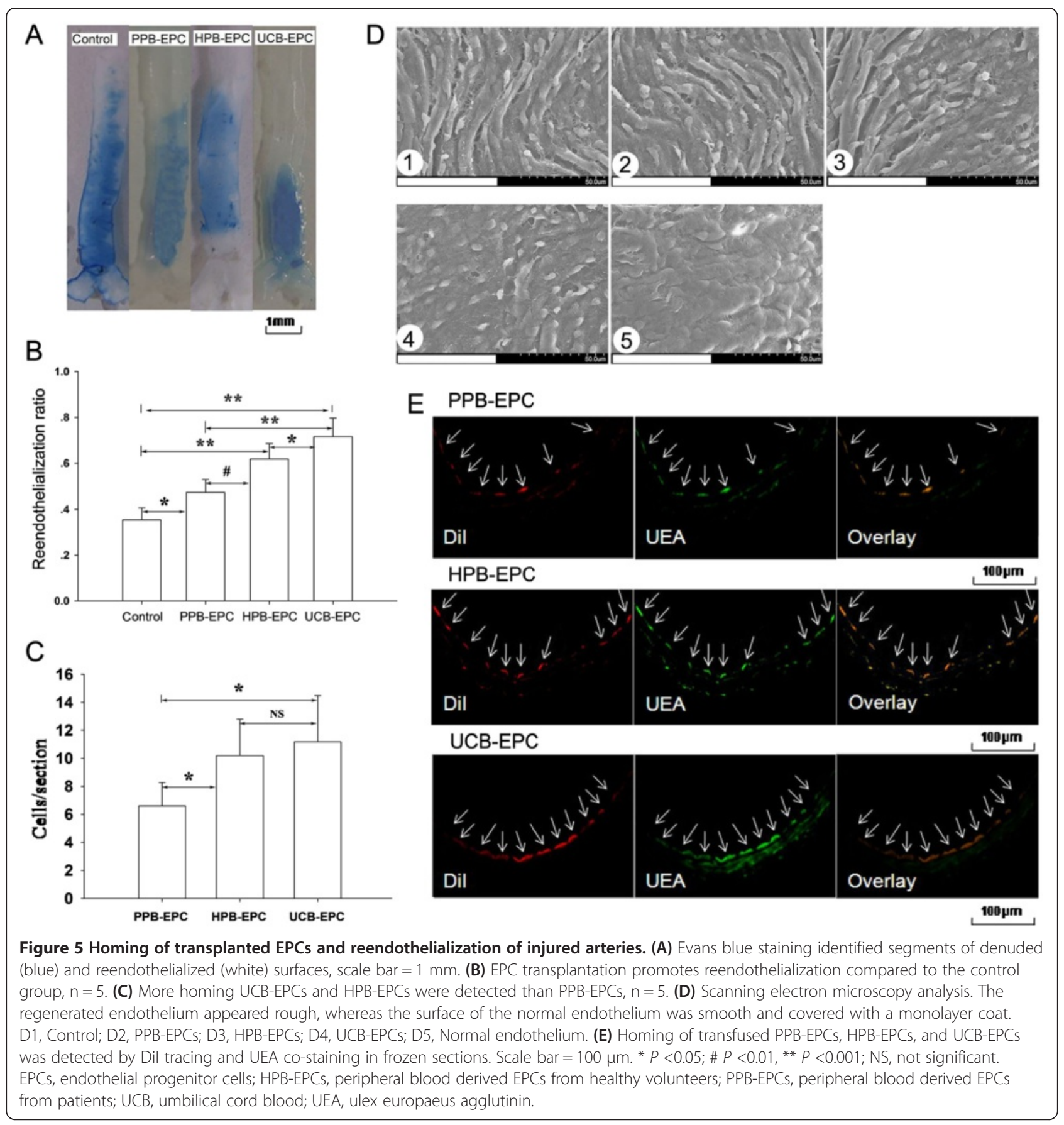

$(P<0.01$ or $P<0.05)$. There was no difference in the neointima area or the neointima/media ratio between the cryopreserved UCB and HPB groups.

\section{Discussion}

In the present study, we report that the transplantation of cryopreserved UCB-EPCs induces the recovery of carotid artery injury in nude rats, indicating a promising strategy for clinical therapy of patients with multiple cardiovascular risk factors.
The integrity and functional activity of the endothelial monolayer plays a crucial role in the prevention of atherosclerosis, a common disease in our aging world population. It is well known that EPCs can home to the injured zone, differentiate into endothelial cells, and promote reendothelialization. However, impaired quantity and quality of these vasculogenic cells in older patients, especially with cardiovascular risk factors, such as age, smoking, hypertension, hyperlipidemia and diabetes, may account for the deterioration of endothelial 


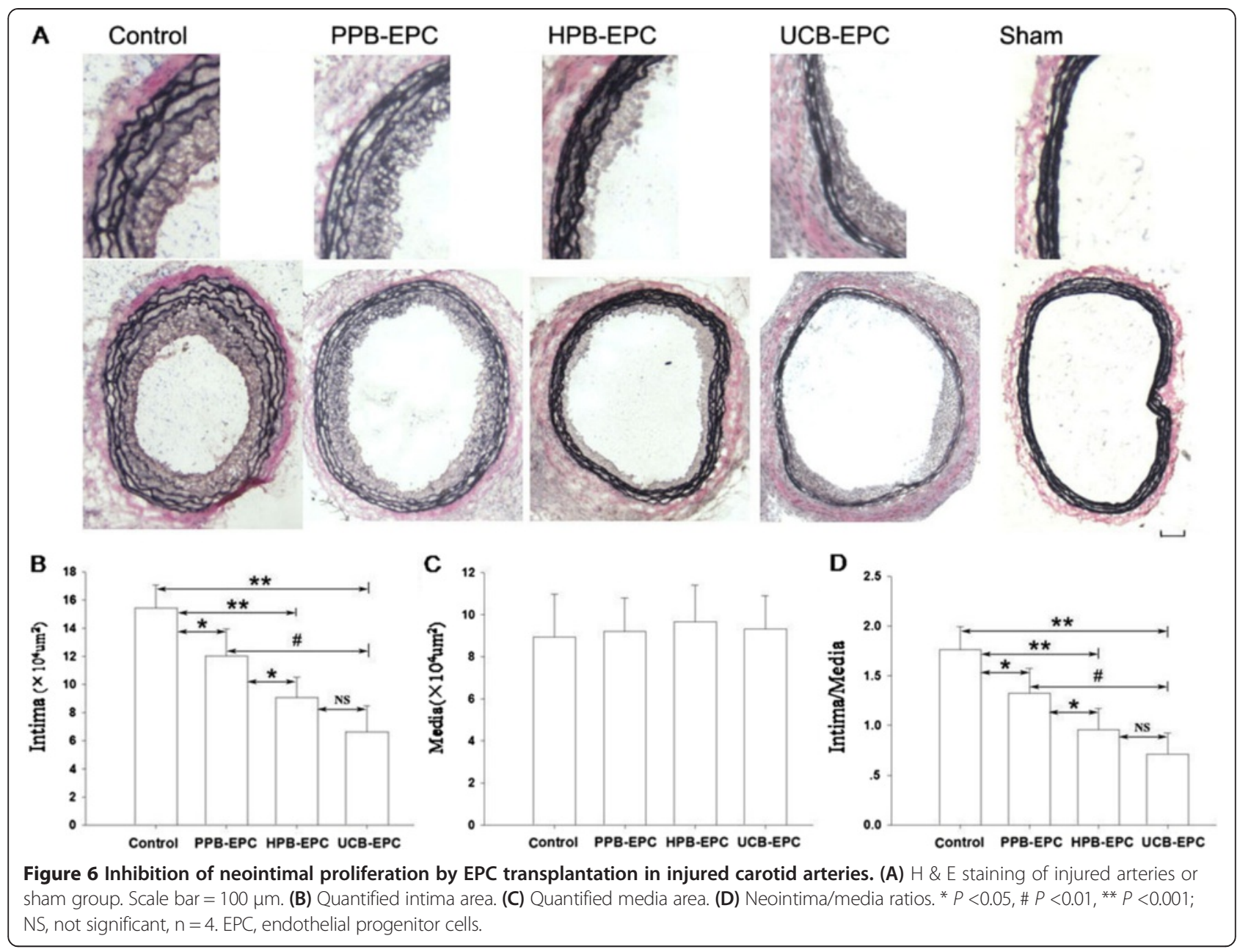

regeneration $[17,18]$. In our study, we also found that EPCs from patients with diabetes, hypertension, coronary artery disease, or aging are associated with impaired migration, proliferation, adhesion, and survival capabilities. Although there is no difference in VEGF and SDF-1 release, our in vivo study showed that infusion of dysfunctional peripheral EPCs led to decreased recovery of carotid artery injury than healthy EPCs.

Previous studies have shown that the transplantation of UCB-EPCs represents a promising therapeutic strategy for multiple cardiovascular diseases [19]. In 2007, it was reported that EPCs could be isolated from frozen $\mathrm{UCB}$ mononuclear cells stored at $-196^{\circ} \mathrm{C}$ for 0.2 to 5 years and differentiated into endothelial cells [9], suggesting that ex vivo culture is a useful method for collection of EPCs from cryopreserved UCB-derived MNCs. Other studies also found that the 'late' EPCs from cryopreserved MNCs were phenotypically and functionally indistinguishable from freshly isolated ones, including the ability to form blood vessels in vivo $[10,11]$. Thus, private collecting of cryopreserved UCB units may provide large quantity and high quality EPCs for personal autotransplantation or matched-patient allotransplantation.

We successfully obtained cluster-forming EPCs from cryopreserved UCB-MNCs, which, based on the time and appearance, seem like late EPCs [20,21]. These progenitors have the capability of tube formation, UEA-binding, Ac-LDL (acetylated low-density lipoprotein) uptake, and expression of endothelial membrane antigens. Furthermore, we also identified Weibel-Palade bodies, the specific ultrastructure of endothelial cells, using electron micrographs.

EPC-mediated paracrine signaling has been shown to be critical in reendothelialization and neovascularization by activating adjacent endothelial cells and enhancing angiogenesis [22,23]. Thus, we checked the secretion of cytokines in the culture medium and found a significant increase in the expression of VEGF and SDF-1 during EPC differentiation.

EPCs can be obtained from MNCs and from purified populations of CD34-positive, CD133-positive, or CD14-positive hematopoietic cells [2,20,21,24]. However, $\mathrm{Lu}$ et al. found that they were unable to generate endothelial-like cells from cryopreserved cord blood (CB) 
by CD34 magnetic cell sorting [25], which in our opinion may be due to the different isolation method they used.

Moreover, Jun-ho et al. compared the potential of cryopreserved UCB-EPCs with those from healthy volunteers' fresh PB (HPB). They found no difference in VEGF secretion and proliferation between the two groups [9]. We found that UCB-EPCs and HPB-EPCs showed a higher capacity for migration, proliferation, survival, and adherence than PPB-EPCs, indicating the well preserved potential of cryopreserved UCB-EPCs. Nevertheless, similar membrane antigen and cytokines, such as VEGF and SDF-1, were expressed with PPB-EPCs.

Absence or delay of reendothelialization is the initial step for thrombosis and neointimal hyperplasia, which leads to in-stent restenosis and subsequently tissue ischemia. However, few studies have been performed to evaluate the availability of cryopreserved autologous UCB-EPCs for transplantation. In this study, DiI-labeled EPCs were located in the injured site, and bound to the UEA specific endothelial marker, indicating differentiated endothelial cells. We also found that the transplantation of cryopreserved UCB-EPCs, PPB-EPCs, and HPB-EPCs increased reendothelialization of the denuded vessels and reduced neointima formation. Furthermore, compared with the PPB group, more homing EPCs were observed in the cryopreserved UCB and HPB groups, which may have subsequently resulted in accelerated reendothelialization and reduced neointimal formation.

\section{Conclusions}

These results show that cryopreserved UCB-EPCs and HPB-EPCs are functionally stronger in vitro and have a greater effect on vascular repair than 'unhealthy PB-EPCs' after in vivo transplantation. Thus, cryopreservation and delivery of cryopreserved autogenous UCB-EPCs or HPB-EPCs may be a promising vasculoprotective approach for patients with multiple cardiovascular risk factors.

\section{Additional files}

Additional file 1: Dil and UEA double staining of cultured PPB-EPCs and HPB-EPCs. Adherent cells from PPB-MNCs (upper) and HPB-MNCS (lower) bound endothelial-specific lectin (green, A1, A2) and took up acetylated LDL (red, B1, B2). Double-positive cultured MNCs (yellow, $90.9 \pm 11.14 \%$ for PPB-MNCs, $91.04 \pm 8.76 \%$ for HPB-MNCs; C1, C2, $n=5$ ).

Additional file 2: Manual counting of EPCs in the PPB, HPB, and UCB groups. VEGF-induced proliferative activity was confirmed by manual counting of EPCS. The number of cells in the UCB and HPB groups was significantly greater than that in the PPB group. NS, not significant; * $P<0.05$; \# $P<0.01,\left(\times 10^{5} /\right.$ well, $\left.\mathrm{n}=5\right)$.

Additional file 3: Control of Evans blue staining and EPC tracking. (A) Normal carotid artery serves as the negative control. (B) Injured artery (day 0) serves as the positive control. (C) No Dil-labeled cells (red) were identified in uninjured control arteries. (D) There were UEA-positive cells within the intima in uninjured control arteries.

\section{Abbreviations}

Dil-LDL: Dil-labeled acetylated low-density lipoprotein; DMEM: Dulbecco's modified Eagle's medium; DMSO: dimethyl sulfoxide; ELISA: enzyme-linked immunosorbent assay; EPCs: endothelial progenitor cells; FBS: fetal bovine serum; FITC: fluorescein isothiocyanate; HPB-EPCs: peripheral blood derived EPCs from healthy volunteers; HSCs: hematopoietic stem cells;

HUVECs: human umbilical venous endothelial cells; MNCs: mononuclear cells; PB: peripheral blood; PBS: phosphate-buffered saline; PFA: paraformaldehyde; PPB-EPCs: peripheral blood derived EPCs from patients; SDF-1: stromal cell derived factor 1; TNF: tumor necrosis factor; UCB: umbilical cord blood; UEA: FITC-labeled lectin from ulex europaeus agglutinin; VEGF: vascular endothelial growth factor.

\section{Competing interests}

The authors declare that they have no competing interests.

\section{Authors' contributions}

YY performed the acquisition, analysis, and interpretation of data, and drafted the manuscript. LH was responsible for designing the study and data analysis. FW and HL carried out all cell culture experiments and PCR analyses, and performed some of the statistical analyses. $L L$ and MD carried out the in vivo experiments and performed some of the statistical analyses.

$\mathrm{XZ}$ conceived of the study and participated in its design, as well as critical manuscript revision. All authors read and approved the final manuscript.

\section{Authors' information}

Yangguang Yin and Huanyun Liu: Co first authors.

\section{Acknowledgements}

This study was supported by grants from the National Natural Science Foundation of China (No. 81070168 and 81370213) and Natural Science Foundation Project of Chongqing (CSTC, 2009BB5020).

\section{Author details}

'Cardiovascular Department, First People's Hospital of Chong Qing New North Zone, Chongqing 401120, China. ${ }^{2}$ Cardiovascular Disease Research Center, Xinqiao Hospital, Third Military Medical University, Chongqing 400037, China.

Received: 29 October 2014 Revised: 29 October 2014

Accepted: 26 February 2015 Published online: 17 April 2015

\section{References}

1. Verma S, Anderson TJ. Fundamentals of endothelial function for the clinical cardiologist. Circulation. 2002;105:546-9.

2. Asahara T, Murohara T, Sullivan A, Silver M, van der Zee R, Li T, et al. Isolation of putative progenitor endothelial cells for angiogenesis. Science. 1997;275:964-7.

3. Zampetaki A, Kirton JP, Xu Q. Vascular repair by endothelial progenitor cells. Cardiovasc Res. 2008;78:413-21.

4. Heiss C, Keymel S, Niesler U, Ziemann J, Kelm M, Kalka C. Impaired progenitor cell activity in age-related endothelial dysfunction. J Am Coll Cardiol. 2005;45:1441-8.

5. Tepper OM, Galiano RD, Capla JM, Kalka C, Gagne PJ, Jacobowitz GR, et al. Human endothelial progenitor cells from type II diabetics exhibit impaired proliferation, adhesion, and incorporation into vascular structures. Circulation. 2002;106:2781-6.

6. Zhu S, Malhotra A, Zhang L, Deng S, Zhang T, Freedman NJ, et al. Human umbilical cord blood endothelial progenitor cells decrease vein graft neointimal hyperplasia in scid mice. Atherosclerosis. 2010;212:63-9.

7. Au P, Daheron LM, Duda DG, Cohen KS, Tyrrell JA, Lanning RM, et al. Differential in vivo potential of endothelial progenitor cells from human umbilical cord blood and adult peripheral blood to form functional long-lasting vessels. Blood. 2008;111:1302-5.

8. Ingram DA, Mead LE, Tanaka H, Meade V, Fenoglio A, Mortell K, et al. Identification of a novel hierarchy of endothelial progenitor cells using human peripheral and umbilical cord blood. Blood. 2004;104:2752-60.

9. Jang JH, Kim SK, Choi JE, Kim YJ, Lee HW, Kang SY, et al. Endothelial progenitor cell differentiation using cryopreserved, umbilical cord blood-derived mononuclear cells. Acta Pharmacol Sin. 2007;28:367-74. 
10. Lin RZ, Dreyzin A, Aamodt K, Dudley AC, Melero-Martin JM. Functional endothelial progenitor cells from cryopreserved umbilical cord blood. Cell Transplant. 2011;20:515-22.

11. Vanneaux V, El-Ayoubi F, Delmau C, Driancourt C, Lecourt S, Grelier A, et al. In vitro and in vivo analysis of endothelial progenitor cells from cryopreserved umbilical cord blood: are we ready for clinical application? Cell Transplant. 2010;19:1143-55.

12. Zhao X, Huang L, Yin Y, Fang Y, Zhao J, Chen J. Estrogen induces endothelial progenitor cells proliferation and migration by estrogen receptors and pi3k-dependent pathways. Microvasc Res. 2008;75:45-52.

13. Zhao $X$, Huang L, Yin Y, Fang Y, Zhou Y. Autologous endothelial progenitor cells transplantation promoting endothelial recovery in mice. Transpl Int. 2007:20:712-21.

14. Yin $Y$, Huang L, Zhao X, Fang Y, Yu S, Zhao J, et al. Amd3100 mobilizes endothelial progenitor cells in mice, but inhibits its biological functions by blocking an autocrine/paracrine regulatory loop of stromal cell derived factor-1 in vitro. J Cardiovasc Pharmacol. 2007:50:61-7.

15. Chen TG, Zhong ZY, Sun GF, Zhou YX, Zhao Y. Effects of tumour necrosis factor-alpha on activity and nitric oxide synthase of endothelial progenitor cells from peripheral blood. Cell Prolif. 2011;44:352-9.

16. Cui B, Huang L, Fang Y, Guo R, Yin Y, Zhao X. Transplantation of endothelial progenitor cells overexpressing endothelial nitric oxide synthase enhances inhibition of neointimal hyperplasia and restores endothelium-dependent vasodilatation. Microvasc Res. 2011:81:143-50.

17. Hill JM, Zalos G, Halcox JP, Schenke WH, Waclawiw MA, Quyyumi AA, et al. Circulating endothelial progenitor cells, vascular function, and cardiovascular risk. N Engl J Med. 2003;348:593-600.

18. Rauscher FM, Goldschmidt-Clermont PJ, Davis BH, Wang T, Gregg D, Ramaswami $\mathrm{P}$, et al. Aging, progenitor cell exhaustion, and atherosclerosis. Circulation. 2003;108:457-63.

19. Zhang L, Yang R, Han ZC. Transplantation of umbilical cord blood-derived endothelial progenitor cells: a promising method of therapeutic revascularisation. Eur J Haematol. 2006;76:1-8.

20. Hur J, Yoon CH, Kim HS, Choi JH, Kang HJ, Hwang KK, et al. Characterization of two types of endothelial progenitor cells and their different contributions to neovasculogenesis. Arterioscler Thromb Vasc Biol. 2004;24:288-93.

21. Lin Y, Weisdorf DJ, Solovey A, Hebbel RP. Origins of circulating endothelial cells and endothelial outgrowth from blood. J Clin Invest. 2000;105:71-7.

22. Rehman J, Li J, Orschell CM, March KL. Peripheral blood "endothelial progenitor cells" are derived from monocyte/macrophages and secrete angiogenic growth factors. Circulation. 2003;107:1164-9.

23. Urbich C, Aicher A, Heeschen C, Dernbach E, Hofmann WK, Zeiher AM, et al. Soluble factors released by endothelial progenitor cells promote migration of endothelial cells and cardiac resident progenitor cells. J Mol Cell Cardiol. 2005;39:733-42.

24. Fernandez Pujol B, Lucibello FC, Gehling UM, Lindemann K, Weidner N, Zuzarte ML, et al. Endothelial-like cells derived from human cd14 positive monocytes. Differentiation. 2000:65:287-300.

25. Lu X, Proctor SJ, Dickinson AM. The effect of cryopreservation on umbilical cord blood endothelial progenitor cell differentiation. Cell Transplant. 2008:17:1423-8.

\section{Submit your next manuscript to BioMed Central and take full advantage of:}

- Convenient online submission

- Thorough peer review

- No space constraints or color figure charges

- Immediate publication on acceptance

- Inclusion in PubMed, CAS, Scopus and Google Scholar

- Research which is freely available for redistribution 